\title{
Refractory Malignant Endocrine Neoplasm
}

National Cancer Institute

\section{Source}

National Cancer Institute. Refractory Malignant Endocrine Neoplasm. NCI Thesaurus.

Code C150541.

Malignant endocrine neoplasm that is resistant to treatment. 